\title{
Estrutura dos Valores Pessoais: A Relação entre Valores Gerais e Laborais
}

\author{
Juliana Barreiros Porto ${ }^{1}$ \\ Centro Universitário Unieuro \\ Universidade Presbiteriana Mackenzie \\ Álvaro Tamayo \\ Universidade de Brasília \\ Universidade Católica de Brasília
}

\begin{abstract}
RESUMO - As pessoas apresentam uma estrutura de valores que guia a sua vida de maneira geral. Essa estrutura é ampla e inclusiva, mas, para focos específicos, existe uma estrutura particular que está relacionada com aquela estrutura mais abrangente. O objetivo deste trabalho foi testar a relação entre as estruturas de valores pessoais gerais e laborais. Para isso, 995 estudantes universitários de ambos os sexos com média de idade de 21 anos responderam às escalas de Valores de Schwartz (SVS) e de Valores relativos ao Trabalho. Os resultados das equações estruturais (AMOS) apoiaram a teoria que relaciona as estruturas de valores $(\mathrm{GFI}=0,95, \mathrm{RMR}=0,04)$. Entretanto, as relações de compatibilidade e conflito foram parcialmente confirmadas. Essa pesquisa responde à necessidade de integração dos modelos de valores gerais com os modelos de valores laborais.
\end{abstract}

Palavras-chave: valores pessoais; valores do trabalho; equações estruturais.

\section{Personal Values Structure: The Relationship between Life and Work Values}

\begin{abstract}
People develop a value structure that guides their lives in general. This structure is wide and inclusive, but, for specific focuses, there is a particular structure that is related to one more extensive. The objective of the present study was to test the relationship between the structures of labor and general personal values. To accomplish this purpose, 995 university students of both sexes and average aged 21 years old answered to Schwartz Value Survey and the Work Values Scale. The results of structural equations (AMOS) supported the theory that relates to the value structures (GFI=0,95, RMR=0,04). However, the conflict and compatibility relations were partially confirmed. This study indicates the necessity to integrate general values with labor values models.
\end{abstract}

Key words: personal values; work values; structural equations.

Schwartz e Bilsky (1987) definem valores como “( $a$ ) princípios ou crenças, (b) sobre comportamentos ou estados de existência, (c) que transcendem situações específicas, (d) que guiam a seleção ou avaliação de comportamentos ou eventos e (e) que são ordenados por sua importância" (p. 551). Os valores guiam as atitudes e o comportamento das pessoas e eles podem estar relacionados a focos específicos da vida do indivíduo, formando estruturas inter-relacionadas. As pessoas apresentam uma estrutura de valores que guia a sua vida de maneira geral. Essa estrutura é ampla e inclusiva, mas, para focos específicos como o trabalho, as pessoas apresentam uma estrutura de valores específica que está relacionada com aquela estrutura mais abrangente (Sagie \& Elizur, 1996).

A maneira como as estruturas de valores se relaciona permanece uma questão em aberto. Poucos estudos têm se detido nessa questão. Entretanto, na área de valores laborais, podem-se ressaltar alguns esforços nessa direção. Elizur e

1 Endereço: SEPS 714/914 Bloco E, Ed. Talento, Sala 107, Brasília, DF, Brasil 70390-145.E-mail: porto.juliana@gmail.com
Sagie (1999) afirmam que analisar os valores pessoais em um arcabouço que considera, simultaneamente, os valores gerais e os valores laborais pode ajudar a clarificar sua importância para o bem-estar pessoal. Os valores laborais são definidos como

princípios ou crenças sobre metas ou recompensas desejáveis, hierarquicamente organizados, que as pessoas buscam por meio do trabalho e que guiam as suas avaliações sobre os resultados e contexto do trabalho, bem como o seu comportamento no trabalho e a escolha de alternativas de trabalho (Porto \& Tamayo, 2003, p. 146).

Nos anos 1960, a relação entre valores gerais e valores laborais já ocupava os estudiosos. Kinnane e Gaubinger (1963), atentos ao problema, realizaram um estudo para testar a hipótese de que os valores da vida têm correlação positiva e significativa com os valores laborais. O Inventário de Valores Laborais de Super (citado por Kinnane \& Gaubinger, 1963) e o Estudo de Valores de Allport-Vernon-Lindzey foram aplicados em 143 estudantes universitários. Os resultados apontaram para uma correlação entre os valores gerais e 
do trabalho com nível de significância inferior a 0,01 , no entanto, os coeficientes foram baixos. Os autores mostraram que quando as pessoas são solicitadas a responder à escala de valores gerais, as suas respostas estão fora de contexto. Assim, teoricamente, obtém-se uma medida pura dos valores. No entanto, quando solicitados a responder à escala de valores laborais, a situação abstrata desaparece e as respostas passam a ser contextualizadas.

Ao se analisar as bases de dados internacionais como Proquest e Ebsco, observa-se que nos anos seguintes o tema deixa de ser investigado, voltando à tona nos anos 1990 com as pesquisas de Elizur e Sagie (1999) e Ros, Schwartz e Surkiss (1999). Elizur e Sagie investigaram a relação entre valores gerais e valores laborais, aplicando em 165 israelenses a Escala de Valores Laborais desenvolvida por Elizur (1991) e mais 21 itens sobre valores gerais elaborados para aquele estudo. Os resultados das análises das distâncias mínimas indicaram uma configuração em formato de cone para a relação entre os valores gerais e do trabalho. Os valores gerais apresentaram-se mais espalhados no espaço multidimensional do que os valores laborais, o que reflete o caráter mais genérico dos valores gerais e mais específico dos valores laborais. Apesar da semelhança entre as estruturas dos valores gerais e do trabalho encontrada no estudo, as diferenças em relação à importância relativa dos itens, individualmente, foram consideráveis. Segundo os autores, essas diferenças indicam que a importância dada aos valores pessoais não é descontextualizada, ao contrário, ela depende do ambiente no qual esses valores são considerados. Os autores concluem que uma visão mais holística, integrando valores laborais e outros valores, pode ser mais produtiva para a compreensão do fenômeno.

O estudo de Elizur e Sagie (1999) ressalta ainda que a relação entre valores gerais e valores específicos, como os valores laborais, pode dar-se de duas maneiras, ou por difusão ou por compensação. No caso de difusão, o que é valorizado de maneira geral também é valorizado nas áreas específicas da vida. Por exemplo, se a pessoa valoriza o sucesso pessoal na sua vida, ela buscará no trabalho, alcançar o sucesso profissional. No caso de compensação, o que é valorizado em um âmbito não o é em outro. Por exemplo, se a pessoa valoriza novidades e desafios em sua vida, no trabalho ela buscaria segurança. As pesquisas têm oferecido apoio para a hipótese de difusão. No entanto, Elizur e Sagie sugerem que esse tópico deve ser objeto de investigação.

Ros e cols. (1999) examinaram a associação entre valores gerais e valores laborais. A partir do modelo de Schwartz para os valores individuais, os autores hipotetizaram que os tipos motivacionais de segunda ordem estariam associados de forma difusa com os valores laborais, assim, Valores Intrínsecos estariam associados positivamente com Abertura à Mudança, Valores Extrínsecos com Conservação, Valores Sociais com Autotranscendência e Valores de Prestígio com Autopromoção. Participaram do estudo 999 judeus de Israel. Os participantes responderam à Escala de Valores de Schwartz (1992) e ao instrumento de valores laborais desenvolvido e validado para o estudo. Os resultados das correlações apresentaram suporte parcial para a hipótese de que valores gerais estariam relacionados com os valores laborais da maneira hipotetizada. Foram encontradas corre- lações: 1) positiva entre Valores Extrínsecos e Conservação e negativa com Abertura à Mudança; 2) positiva entre Valores Intrínsecos e Abertura à Mudança e negativa com Conservação; 3) positiva entre Valores Sociais e Autotranscendência e negativa com Autopromoção; 4) positiva entre Valores de Prestígio e Autopromoção e negativa com Autotranscendência. Porém, os Valores Sociais e de Prestígio correlacionaram-se também com a outra dimensão bipolar (Abertura à Mudança e Conservação) devendo ser esses resultados investigados em estudos futuros.

Em suma, fica evidente a preocupação das pesquisas atuais em integrar as teorias de valores laborais às teorias mais amplas de compreensão dos valores e investigar a relação entre valores gerais e do trabalho. Os trabalhos têm apresentado resultados na direção da integração, porém ainda é preciso dedicar esforços para a compreensão da relação entre as estruturas de valores e verificar a pertinência das estruturas propostas em outros países.

Assim, o objetivo deste artigo é investigar a relação entre a estrutura dos valores pessoais e a estrutura dos valores laborais, buscando testar as relações previstas em estudos anteriores por meio de equações estruturais. As teorias que embasam este estudo são a Teoria de Valores Schwartz e a Teoria de Valores Laborais de Ros e cols. (1999), que serão apresentadas a seguir.

\section{Teoria dos Valores Humanos}

Para Schwartz e Bilsky (1987), os valores são "representações cognitivas de três tipos de necessidades humanas universais: necessidades biológicas do organismo, necessidade de interação social para a regulação das relações interpessoais e necessidades sócioinstitucionais, que visam o bem-estar e sobrevivência do grupo" (p.551).

Schwartz (1992), após revisão da estrutura inicialmente proposta por Schwartz e Bilsky (1987, 1990), propôs uma tipologia universal de valores baseada na literatura sobre as necessidades básicas dos indivíduos. Os tipos motivacionais por eles propostos foram: Universalismo (Compreensão e proteção do bem-estar de todos e da natureza), Benevolência (Preocupação com o bem-estar de pessoas próximas), Conformidade (Restrição de ações e impulsos que podem magoar outros ou violar as expectativas sociais e normas), Tradição (Respeito, compromisso e aceitação dos costumes e idéias de uma cultura ou religião), Segurança (Segurança, harmonia e estabilidade da sociedade, dos relacionamentos e do self), Poder (Preservação de uma posição social dentro de um sistema social), Realização (Procura do sucesso pessoal por meio de demonstração de competência de acordo com as regras sociais), Hedonismo (Prazer e gratificação para si mesmo) e Estimulação (Novidade e estimulação na vida).

O modelo teórico de Schwartz (1992) também prevê uma estrutura dinâmica entre os tipos motivacionais de forma que as pessoas tendam a apresentar alta prioridade para tipos motivacionais mutuamente compatíveis e baixa prioridade para tipos motivacionais conflitivos. Portanto, a prioridade dada aos tipos motivacionais não se estabelece de forma aleatória, mas de forma coerente com os domínios motivacionais. Para os autores, a priorização simultânea de alguns domínios incompatíveis levaria a conflitos. 
Esses 10 tipos motivacionais podem ser classificados em quatro tipos motivacionais de segunda ordem (Schwartz, 2005): 1) Autopromoção - agrega Poder, Hedonismo e Realização; 2) Autotranscendência - agrupa Universalismo e Benevolência; 3) Abertura à Mudança - agrega Hedonismo, Estimulação e Autodeterminação; 4) Conservação - inclui Segurança, Conformidade e Tradição. Como se pode constatar na classificação, o tipo "Hedonismo" participa de duas dimensões de ordem superior.

Os tipos motivacionais de segunda ordem representam as relações de compatibilidade dentro de cada agrupamento e também as relações de conflito entre eles. Dessa maneira, formam duas dimensões bipolares: Autopromoção versus $\mathrm{Au}-$ totranscendência e Abertura à Mudança versus Conservação (Schwartz, 2005). A primeira dimensão contrasta a busca de sucesso pessoal e poder sobre os outros com a busca pelo bem-estar dos outros. A segunda dimensão opõe a ênfase na independência de ação e pensamento à auto-restrição que promove a preservação da estabilidade.

Schwartz (2005) desenvolveu uma estrutura circular para representar a sua teoria ressaltando os tipos motivacionais, os fatores de segunda ordem e as relações de compatibilidade e conflito. Na estrutura, quanto mais próximo um tipo motivacional estiver de um outro, maior a probabilidade de compatibilidade e quanto mais afastado, maior a de conflito (Figura 1).

Estudos transculturais (Schwartz, 2005) têm demonstrado a consistência dessa teoria. Esses estudos levam à conclusão de que os 10 tipos motivacionais têm surgido nas análises feitas em diferentes culturas. Os estudos também comprovam que a adição de valores específicos a cada cultura não tem modificado a estrutura apresentada, novos valores incluídos têm sido estatisticamente agregados a um tipo motivacional já existente.

Recentemente, Gouveia, Martínez, Meira e Milfont (2001) testaram a estrutura e o conteúdo da tipologia de Schwartz (1992) por meio de análise fatorial confirmatória. Os resultados permitiram concluir que a teoria é adequada, apesar de os índices de adequação do modelo não serem excepcionais (GFI $=0,87$ e RMSR $=0,08$ ). Foram confirmadas

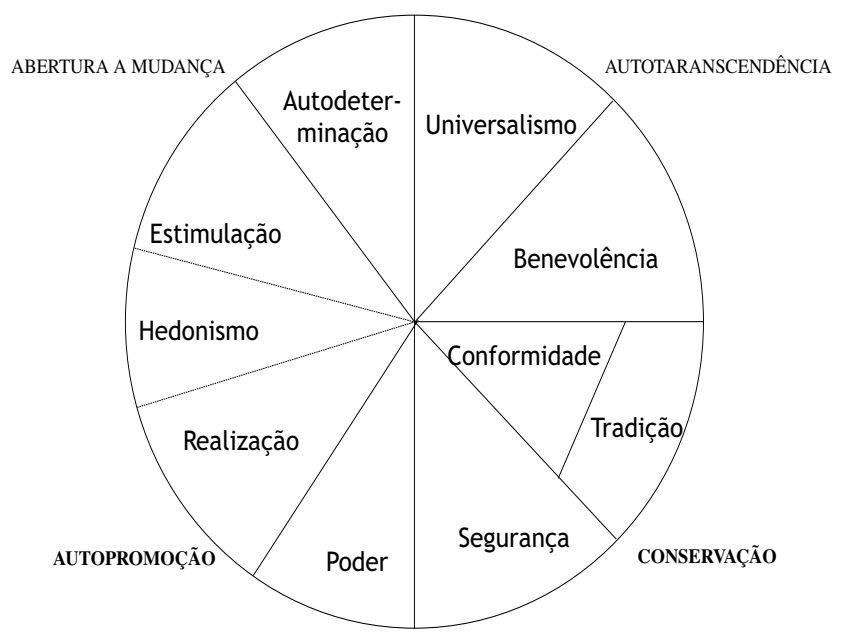

Figura 1. Estrutura Circular dos Valores segundo Schwartz (1992). as relações de compatibilidade, mas as relações de conflito foram apenas parcialmente confirmadas.

Schwartz e Boehnke (2004) também realizaram um teste do modelo por meio da análise fatorial confirmatória, mas eles usaram um desenho específico para isso, utilizando o LISREL. Os seus resultados apontam para a confirmação da teoria, inclusive das relações de conflito entre os tipos motivacionais. Eles sugerem que o modelo quase-cincumplex, em que o tipo motivacional de tradição assume uma posição periférica em relação à conformidade, é o mais adequado. Os resultados dão suporte para se trabalhar com as dimensões bipolares. Um resultado que acrescenta ao modelo teórico refere-se ao teste da relação de hedonismo com os dois fatores de ordem superior "Abertura à mudança" e "Autopromoção". Os dados apontam que Hedonismo relaciona-se mais fortemente com "Abertura à mudança". Assim, hedonismo está focado mais na busca "livre das experiências de prazer e menos na busca competitiva de prazer" (p. 252).

\section{Teoria de Valores Laborais de Ros, Schwartz e Surkiss}

A partir da constatação da lacuna nas pesquisas sobre valores laborais, as quais não têm considerado o desenvolvimento teórico da área de valores, Ros e cols. (1999) revisam as estruturas sobre valores laborais presentes na literatura e sugerem um modelo que se baseia na Teoria de Schwartz dos Valores Pessoais.

Segundo a teoria de Schwartz, podemos identificar quatro tipos motivacionais de segunda ordem: Autotranscendência, Autopromoção, Abertura à Mudança e Conservação. Considerando os valores laborais como expressões dos valores gerais no ambiente de trabalho, podemos supor que os quatros tipos motivacionais propostos na teoria dos valores gerais podem ser encontrados para os valores laborais. Ros e cols. (1999) analisaram a literatura e identificaram que os modelos anteriores apresentavam três dos quatro tipos propostos: 1) Valores Intrínsecos, que estariam associados à Abertura à Mudança; 2) Valores Extrínsecos ou materiais associados à Conservação; e 3) Valores Sociais ou Afetivos associados à Autotranscendência. Porém, o tipo motivacional de Autopromoção não consta como uma dimensão dos valores laborais, apesar de itens que o representam estarem presentes nos modelos anteriores, tais como prestígio, autoridade, influência e poder. Os autores reexaminaram os resultados de Elizur (1991) e indicaram que a distinção desse quarto tipo poderia tornar o modelo mais claro. Da mesma forma que nos valores gerais, era esperada a dinâmica de compatibilidade e conflito entre os valores laborais.

Os autores, portanto, apontam para quatro tipos de valores laborais: 1) Intrínseco - refere-se a metas obtidas pelo conteúdo do próprio trabalho; 2) Extrínseco - refere-se a metas obtidas pelos resultados do trabalho; 3) Social - refere-se à busca de metas relacionais e; 4) Prestígio - refere-se à busca de poder e prestígio por meio do trabalho.

A análise do instrumento desenvolvido pelos autores indicou a presença dos quatro fatores tanto por meio da SSA quanto da análise de componentes principais, sendo que os valores extrínsecos estavam em oposição aos intrínsecos e os valores sociais em oposição aos de prestígio. 
Outra pesquisa que integrou a teoria de Schwartz ao estudo dos valores laborais foi desenvolvida no Brasil por Borges (1998, 1999). Ela elaborou o Inventário do Significado do Trabalho (IST) distinguindo duas classes de atributos: valorativos e descritivos. Segundo Borges (1999), os atributos valorativos são os valores laborais e "consistem em uma definição do que 'deve ser' o trabalho" (p.110). Esse Inventário tem a vantagem de investigar aspectos característicos da cultura brasileira e também a de considerar aspectos da corrente marxista relativos ao trabalho como exploração, hominização, embrutecimento e alienação. Além disso, o IST pode ser aplicado em populações sem alfabetização ou com baixa escolaridade.

Seguindo a perspectiva de integração das teorias de valores gerais às de valores laborais, a autora comparou os resultados encontrados à teoria de Schwartz. Para Borges, a teoria de Schwartz dá apoio aos dados. A seguir, são apresentados os cinco fatores encontrados por meio da análise fatorial do instrumento sobre atributos valorativos e a relação de cada um deles com a estrutura de Schwartz (2005): 1) Exigências sociais: refere-se à semelhança e repetição das tarefas e à contribuição ao progresso social. Esse fator está associado aos tipos motivacionais de Tradição, Conformidade e Universalismo; 2) Justiça no trabalho: agrega itens que definem o trabalho com a função de proteger o indivíduo. Fator associado à Segurança e Universalismo; 3) Esforço corporal e desumanização: refere-se à opinião de que o trabalho deve representar esforço corporal. Fator distinto, sem correspondência como o modelo de Schwartz; 4) Realização pessoal: reúne itens sobre o prazer associado ao trabalho. Valores esses associados ao Hedonismo e Realização; 5) Sobrevivência pessoal e familiar: refere-se ao papel do trabalho de garantir sustento ao indivíduo e à família. Fator associado à Autodeterminação.

As análises de ordem superior revelaram dois fatores denominados Sócioexigências e Humanização. O primeiro representa as exigências da sociedade e o segundo as expectativas do indivíduo em relação às condições almejadas para atender as primeiras. Essa estrutura representa as dimensões opostas entre Individualismo e Coletivismo.

Com base no modelo teórico de Ros e cols. (1999), Porto e Tamayo (2003) desenvolveram e validaram uma escala de valores laborais no Brasil. Para a construção da escala, foram pesquisados instrumentos publicados na literatura e entrevistados trabalhadores. Os resultados da análise fatorial indicaram os mesmos quatro fatores encontrados por Ros e cols. e foram assim denominados: 1) Realização Profissional: refere-se à busca de prazer e realização pessoal e profissional, bem como de independência de pensamento e ação no trabalho por meio da autonomia intelectual e da criatividade; 2) Estabilidade: refere-se à busca de segurança e ordem na vida por meio do trabalho, possibilitando suprir materialmente as necessidades pessoais; 3) Relações Sociais: refere-se à busca de relações sociais positivas no trabalho e de contribuição positiva para a sociedade por meio do trabalho; 4) Prestígio: refere-se à busca de autoridade, sucesso profissional e poder de influência no trabalho.

A partir dos trabalhos relatados pode-se supor que a estrutura de valores laborais relaciona-se com a estrutura de valores gerais. Todavia, não foram realizados testes empíricos que relacionassem essa estrutura à dos valores pessoais no país.

Teoricamente, o modelo de Ros e cols. (1999) está associado ao modelo de Schwartz (2005). A dimensão Abertura à Mudança e Conservação de Schwartz está associada à dimensão Realização Profissional e Estabilidade, enquanto a dimensão Autotranscendência e Autopromoção às Relações Sociais e Prestígio. Assim, derivam-se as seguintes hipóteses:

Hipótese 1 - Realização Profissional relacionar-se-á a Abertura à Mudança pela ênfase dada à mudança por meio da autonomia e criatividade;

Hipótese 2 - Relações Sociais estará associado à Autotranscendência pela ênfase em proporcionar o bem-estar da sociedade e das pessoas próximas;

Hipótese 3 - Prestígio estará associado à Autopromoção pela importância dada à influência e sucesso pessoal;

Hipótese 4 - Estabilidade relacionar-se-á a Conservação pela busca de segurança e manutenção do status quo.

Além disso, os modelos de Ros e cols. (1999) e Schwartz (2005) apresentam as duas dimensões Abertura à Mudança x Conservação e Autotranscendência x Autopromoção como independentes, e os pólos de cada dimensão expressam valores conflitantes entre si. Dessa forma, geram-se as hipóteses de relações conflitantes entre os pólos.

Hipótese 5 - Realização Profissional se associará negativamente com Conservação, visto que o primeiro busca a independência de pensamento e ação e o último a auto-restrição dos interesses individuais em prol do grupo.

Hipótese 6 - Estabilidade estará associado negativamente com Abertura à Mudança, pois a sua ênfase é na busca de segurança e não na abertura a experiências novas e variadas.

Hipótese 7 - Relações Sociais estará relacionado negativamente com Autopromoção, pois um busca relações sociais positivas com os outros, o que é incompatível com a busca de poder e de promoção de si mesmo.

Hipótese 8 - Prestígio se associará negativamente com Autotranscendência devido a sua ênfase no sucesso pessoal em detrimento da busca do bem-estar das pessoas.

Estudos têm discutido se a relação entre valores laborais e valores gerais é de compensação ou de difusão. Ou seja, se os valores laborais apresentam uma hierarquia em oposição aos valores gerais de maneira a compensar no trabalho aquilo que não é valorizado de maneira geral ou, se pelo contrário, os valores gerais contaminam os valores laborais de tal forma que o que é valorizado no geral também é valorizado no trabalho. A pesquisa de Elizur e Sagie (1999) aponta que há uma relação de difusão. Assim, espera-se demonstrar no presente trabalho que:

Hipótese 9 - Os valores gerais do indivíduo apresentam relação positiva com os valores laborais.

\section{Método}

\section{Amostra}

Foi selecionada uma universidade do Distrito Federal por conveniência para realização da pesquisa. Dezoito cursos foram escolhidos aleatoriamente e os alunos de disciplinas, também escolhidas aleatoriamente, foram convidados a 
participar. No total, 995 estudantes universitários com média de idade de 21 anos $($ d.p. $=4,14)$ responderam o questionário. Dos participantes, $51 \%$ eram do sexo feminino, $92 \%$ eram solteiros, $75 \%$ moravam com os pais, $58 \%$ possuíam alguma experiência de trabalho e $45 \%$ eram católicos.

\section{Instrumento}

No estudo, foram utilizadas duas escalas de valores. A primeira visa avaliar os valores laborais e a segunda os valores gerais.

Escala de Valores relativos ao Trabalho (EVT) - desenvolvida e validada por Porto e Tamayo (2003). A escala é composta por 45 valores laborais que devem ser avaliados por uma escala de cinco pontos que varia de "nada importante" (1) até "muito importante" (5). A análise fatorial para a presente amostra apresentou os mesmos quatro fatores da validação da escala, entretanto, 13 itens foram eliminados por apresentarem índices inferiores a 0,45 ou por não apresentarem equivalência entre os cursos universitários a partir de análises fatoriais procrustes conforme relatado por Porto, Ros e Tamayo (2003). Os quatro fatores encontrados e seus respectivos alphas de Cronbach foram:

Fator 1 - Realização no trabalho. $(\alpha=0,87)$ : Refere-se à busca de prazer e realização pessoal e profissional, bem como de independência de pensamento e ação no trabalho por meio da autonomia intelectual e da criatividade.

Fator 2 - Relações Sociais $(\alpha=0,86)$ : Refere-se à busca de relações sociais positivas no trabalho e de contribuição positiva para a sociedade por meio do trabalho.

Fator 3 - Prestígio $(\alpha=0,85)$ : Refere-se à busca de autoridade, sucesso profissional e poder de influência no trabalho.

Fator 4 - Estabilidade $(\alpha=0,84)$ : Refere-se à busca de segurança e ordem na vida por meio do trabalho, possibilitando suprir materialmente as necessidades pessoais.

Escala de Valores do Schwartz - Os valores gerais foram investigados por meio da Escala de Valores Humanos de Schwartz (1992), traduzida e validada para o Brasil por Tamayo e Schwartz (1993) pela SSA (Guttman, 1968). O instrumento apresenta 61 valores que devem ser avaliados por uma escala de importância que varia de -1 (valores opostos aos meus valores) a 7 (valores de suprema importância). Para a presente amostra, foram calculadas análises de escalonamento multidimensional para agrupamentos por áreas do conhecimento, conforme classificação do Ministério da Educação e Cultura (2002): 1) Ciências Biológicas e Saúde, 2) Ciências Exatas e da Terra, 3) Ciências Humanas e Sociais, 4) Ciências Sociais Aplicadas e 5) Engenharia e Tecnologias. Somente os itens que estavam relacionados à mesma dimensão em todos os grupos é que foram considerados na análise. A Figura 2 apresenta a lista dos itens utilizados na análise. A escala avalia os 10 tipos motivacionais propostos teoricamente por Schwartz (1992): Poder, Realização, Hedonismo, Estimulação, Autodeterminação, Universalismo, Benevolência, Tradição, Conformidade e Segurança. Esses tipos motivacionais podem ser agrupados em quatro facetas de segunda ordem que foram as utilizadas na presente pes- quisa, a saber: Abertura à mudança $(\alpha=0,83)$, Conservação $(\alpha=0,82)$, Autotranscendência $(\alpha=0,85)$ e Autopromoção $(\alpha=0,82)$.

O resultado da análise de escalonamento multidimensional (Proxcal), por meio do Statistical Package for Social Science v.13 para a presente amostra, indicou 10 regiões. No entanto, os tipos motivacionais de Conformidade e Tradição se localizaram lado a lado e não um atrás do outro como previsto pelo modelo teórico. Os índices de adequação do modelo foram: S-Stress=0,27; Stress I=0,36; Stress II=0,89; Coeficiente de congruência de Tucker=0,93. Apesar do S-Stress ter sido abaixo do recomendado por Schiffman, Reynolds e Young (1981), para a definição do número de dimensões optou-se por uma escolha teórica e não estatística como sugere Shye, Elizur e Hoffman (1994). Assim, decidiuse por uma estrutura bidimensional.

Variáveis demográficas e familiares - Além das escalas, os participantes responderam às perguntas sobre as seguintes variáveis: gênero, curso de graduação, ano de entrada no curso, idade, religião, experiência de trabalho, estado civil, estrutura familiar (se o estudante mora ou não com seus pais; se os pais são casados, divorciados ou viúvos; e, se os avós moram na mesma residência), nível de escolaridade dos pais e estado civil dos pais.

\section{Coleta de dados}

Inicialmente, a pesquisa foi apresentada aos coordenadores dos cursos selecionados aleatoriamente e foi solicitada autorização para que o pesquisador contatasse os professores e agendasse com eles a data de aplicação do questionário em sala de aula. Uma vez agendado o encontro com os professores, um membro da equipe de pesquisa convidava em sala os estudantes a participar da pesquisa, explicitando a participação voluntária e a não-identificação dos participantes, conforme previsto pelo projeto aprovado em comitê de ética. Os alunos que aceitaram participar responderam o questionário em sala de aula e o devolveram diretamente ao pesquisador. $\mathrm{O}$ tamanho das turmas variou muito, de oito a 70 alunos, e os respondentes levaram, em média, 30 minutos para concluir a tarefa.

\section{Análise dos dados}

Inicialmente, foi realizado o estudo de sistematicidade dos casos omissos (Little \& Rubin, 1987). Observou-se a ausência de sistematicidade de casos omissos, o que permite o uso do método de imputação simples. Assim, os dados omissos (menos de 2\%) foram substituídos por meio da regressão. Quando o respondente deixou mais de 12 itens sem resposta, o questionário foi eliminado $(n=33)$.

Para investigar as relações entre as estruturas de valores, foram realizadas equações estruturais, utilizando o programa AMOS. Inicialmente, foram realizadas análises fatoriais confirmatórias de cada instrumento e, a seguir, a equação estrutural para verificar a relação entre as estruturas. As análises do grau de assimetria das variáveis e do coeficiente de Mardia (1971) apontaram para a falta de normalidade multivariada tanto da EVT quanto da SVS. Procedeu-se, então, à transformação das variáveis conforme indicações de 
Osborne (2002). Foram realizadas transformações logarítimicas normais e pela raiz quadrada. A normalidade dos dados melhorou, mas as transformações não foram suficientes para eliminar completamente a falta de normalidade. O método de estimação utilizado, portanto, foi o dos Mínimos Quadrados Não-ponderados (ULS), apontado por Bollen (1989) como menos sensível ao efeito da falta de normalidade dos dados. Entretanto, o método não apresenta os testes de significância estatística dos parâmetros estimados (Bollen, 1989). Os índices de ajuste do modelo utilizados foram o GFI, AGFI e RMR. De acordo com Ullman (2001), o GFI e o AGFI são índices de ajuste relativos à proporção de variância explicada pelo modelo em teste e recomendam-se valores acima de 0,90 para aceitação do modelo como adequado aos dados. A mesma autora indica que o RMR é um índice baseado nos resíduos da análise e avalia a distância entre os valores estimados e observados na matriz de covariância de dados. Nesse caso, espera-se um valor próximo a zero como indicador de ajuste próximo.

\section{Resultados}

Os resultados das análises fatoriais confirmatórias das duas escalas de valores apontaram para a boa adequação dos modelos, conforme relato a seguir.

Para a EVT, a razão entre o $\chi^{2}(224,34)$ e os graus de liberdade (458) foi 0,49 (Kline, 1998), indicando a aceitação do modelo. Os demais índices apontaram para uma boa adequação do modelo de quatro fatores ( $\mathrm{RMR}=0,02, \mathrm{GFI}=0,98$, AGFI $=0,98$ ) e nenhuma modificação foi realizada em relação ao modelo teórico, já que os índices GFI e AGFI estão acima de 0,95 e o RMR está abaixo de 0,05 . O coeficiente de Mardia de normalidade multivariada foi de 106,53 (valor crítico $=36,02$ ), indicando falta de normalidade multivariada e a necessidade de se utilizar estimadores menos sensíveis a esse problema.

Para a estrutura de segunda ordem da SVS, a razão entre o $\chi^{2}(3142,73)$ e os graus de liberdade (1218) foi 2,58, indicando a aceitação do modelo (Kline, 1998). Os demais índices apontaram para um bom ajuste $(\mathrm{RMR}=0,05, \mathrm{GFI}=0,97, \mathrm{AGFI}=0,96) \mathrm{e}$ nenhuma modificação foi realizada. O coeficiente de Mardia de normalidade multivariada foi de 432,416 (valor crítico=92,76), indicando falta de normalidade multivariada.

Finalmente, a modelagem por equações estruturais para verificar a relação entre os valores gerais e do trabalho apontou para a aceitação do modelo. A razão entre $\chi^{2}(6390,33)$ e os graus de liberdade (3306) foi 1,93, indicando a aceitação do modelo. Os demais índices revelaram uma boa adequação ( $R M R=0,04$, GFI $=0,95$, AGFI $=0,95$ ). A Figura 2 apresenta o modelo com os parâmetros padronizados estimados pela ULS. Para facilitar a visualização, foram eliminados os errospadrão, os resíduos e as covariâncias entre os fatores. Foram encontradas covariâncias positivas e altas entre os pólos Abertura à mudança e Realização profissional, Conservação e Estabilidade, Autotranscendência e Relações sociais, e Autopromoção e Prestígio. As covariâncias entre as dimensões opostas foram baixas, praticamente nulas, entretanto, entre os pólos de Autopromoção x Relações sociais e Autotranscendência $x$ Prestígio elas seguiram a direção negativa, como previsto teoricamente.

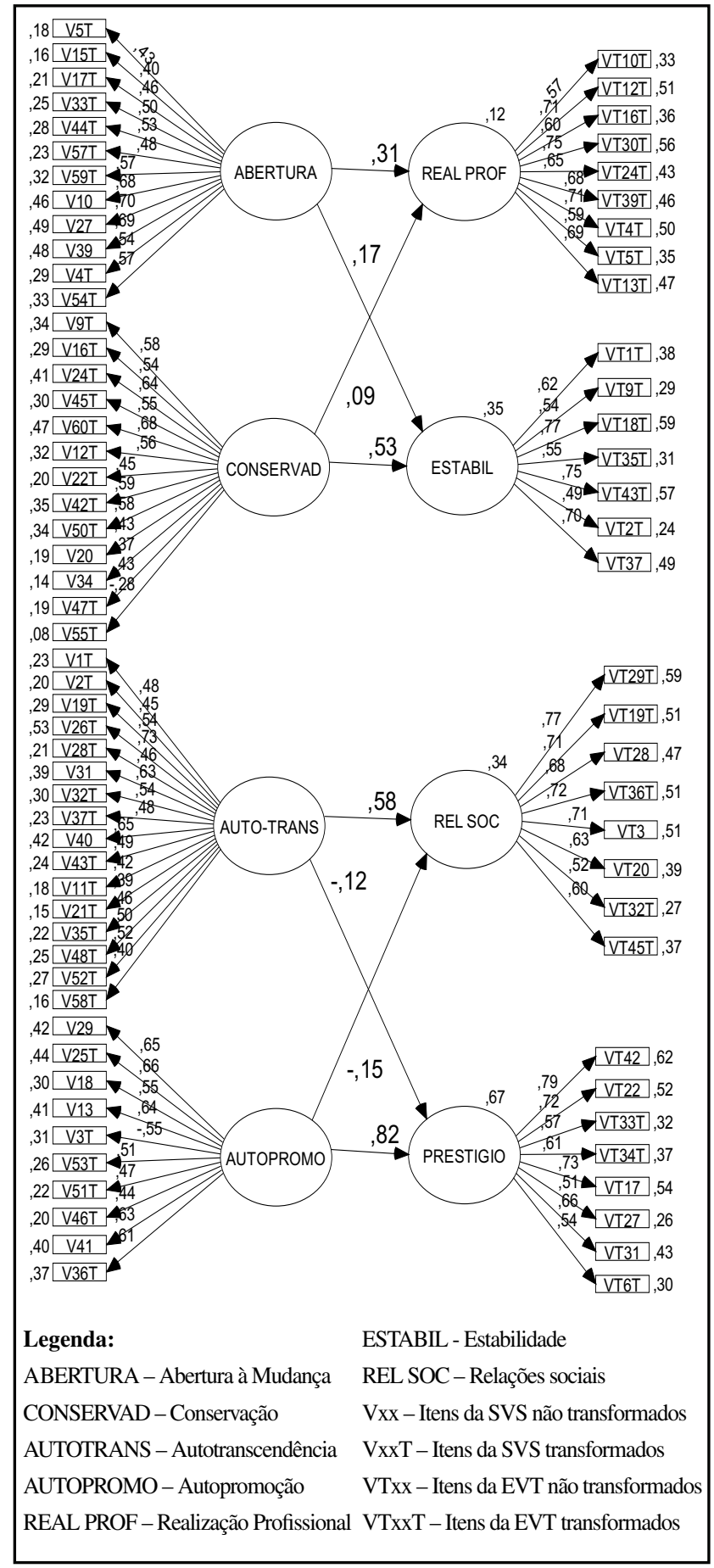

Figura 2. Modelo da Equação Estrutural para a relação entre Valores Gerais e Valores Laborais.

\section{Discussão}

Os resultados apóiam a teoria que aponta para a relação entre as estruturas de valores, já que os índices de ajuste do modelo foram bons. As hipóteses de 1 a 4 , que previam relações positivas entre: 1) Realização Profissional e Abertura à Mudança; 2) Relações Sociais e Autotranscendência; 3) Prestígio e Autopromoção; e 4) Estabilidade e Conservação foram apoiadas pelos dados que apresentaram covariâncias altas entre esses fatores. 
Os resultados fortalecem a teoria de valores humanos, que prevê estruturas inter-relacionadas, ou seja, os indivíduos apresentam uma estrutura geral de valores e, a partir dela, estruturas específicas são formadas para contextos específicos e significativos da vida da pessoa como, por exemplo, o trabalho. Os resultados são congruentes com aqueles encontrados por Elizur (1991) e Ros e cols. (1999).

Com relação às hipóteses de conflito entre os tipos motivacionais, hipóteses de 5 a 8, os resultados apresentam apoio parcial. A hipótese 5, que previa associação negativa entre Realização Profissional e Conservação e a hipótese 6, que previa associação negativa entre Estabilidade e Abertura à Mudança foram refutadas, já que não foram encontradas covariâncias significativas entre essas dimensões. As hipóteses 7 e 8 foram parcialmente confirmadas, pois foram encontradas covariâncias negativas entre Relações Sociais e Autopromoção, e ente Prestígio e Autotranscendência.

A procura por sucesso pessoal e influência social dificulta a busca de relações sociais positivas no trabalho e, principalmente, a contribuição para a sociedade por meio do trabalho, pois o indivíduo que está preocupado em perseguir os seus interesses pessoais dá menos importância aos do grupo social. Devido à ênfase dada ao trabalho na sociedade atual, o ambiente de trabalho é, por excelência, o local para o alcance das metas pessoais de autopromoção, enquanto o outro pólo da dimensão pode ser alcançado em outros contextos sociais como as atividades de voluntariado, participação em ações comunitárias e outras. Além disso, fica evidente que os interesses de autopromoção são incongruentes com a visão do trabalho como instrumento de contribuição positiva para a sociedade.

Em contraposição, a busca do bem-estar de todos e das pessoas próximas dificulta a busca de prestígio no trabalho. Apesar de as metas de autotranscendência poderem ser obtidas em outros contextos, a procura de prestígio no trabalho pode ter um impacto negativo sobre a preocupação com o bem-estar dos outros. A procura de prestígio pode implicar tempo e atenção reduzidos às pessoas próximas, competição com colegas e, às vezes, ações que prejudiquem a sociedade de forma ampla. Dessa forma, o prestígio teria consequiências negativas para a busca da autotranscendência.

Diferentemente do proposto para os valores gerais, no caso dos valores laborais, a relação entre estes tipos motivacionais Abertura à Mudança e Estabilidade e entre Conservação e Realização Profissional não é incompatível. As metas de Abertura à Mudança são compatíveis com a busca de segurança no trabalho, bem como as metas de Conservação são compatíveis com a busca de independência no ambiente de trabalho, apesar dessa última relação ser bastante fraca.

É possível que, para a amostra pesquisada, a estabilidade no trabalho proporcione o alcance do hedonismo, estimulação e autodeterminação, pois somente a partir do momento em que são supridas as necessidades materiais é que se torna possível buscar essas outras metas. Além disso, o fator Estabilidade só possui itens que refletem segurança e não conformidade e tradição que poderiam ser vistos como incompatíveis com as metas de Abertura à Mudança. Assim, ao menos no que diz respeito à segurança, a busca de estabilidade no trabalho não é incompatível com Abertura à Mudança, mas pode ser, inclusive, situação necessária para que a outra se realize. Por exemplo, para que o indivíduo exercite a sua criatividade, pense em coisas novas ou faça coisas diferentes, é importante ter independência financeira e estabilidade no trabalho para se sentir livre e ter condições materiais para perseguir os seus interesses pessoais.

Por outro lado, é importante ressaltar que o estudo de Gouveia e cols. (2001) também não encontrou conflitos em 7 de 12 correlações possíveis para o Inventário de Valores de Schwartz. Os autores argumentam que, na análise fatorial confirmatória, as dispersões podem ser minimizadas em função do agrupamento dos itens e, assim, serem erroneamente interpretadas como ausência de "conflito". Uma solução seria testar a relação entre valores pessoais gerais e valores laborais, utilizando a técnica proposta por Schwartz e Boehnke (2004).

Finalmente, os resultados respondem à necessidade de integração dos modelos de valores gerais com os modelos de valores laborais, contribuindo para a compreensão do sistema de valores dos indivíduos. Os resultados também mostram que o mecanismo de difusão (Elizur, 1991) pode ser o mais apropriado para explicar a relação entre os valores gerais e os valores laborais, conforme a hipótese 9 . Ou seja, os valores gerais guiam os valores pessoais específicos relativos ao mundo do trabalho, sendo que, quanto mais os valores gerais são importantes para a pessoa, mais ela buscará esses valores no contexto do trabalho. Não foi encontrado um mecanismo compensatório em que o que é valorizado na vida em geral seria menos valorizado na vida profissional. É possível, entretanto, que exista um mecanismo compensatório entre os domínios específicos dos valores, por exemplo, entre valores laborais e valores de lazer. Esses resultados apontam para a importância do trabalho no alcance das motivações dos indivíduos, já que o trabalho permite atingir metas que são importantes de maneira geral para as pessoas.

O estudo corroborou a inter-relação entre os valores pessoais gerais e os valores laborais. Os resultados obtidos são coerentes com o trabalho de Sagie e Elizur (1996), que havia apontado a existência de uma estrutura inter-relacionada para os valores de diversos domínios da vida. O presente trabalho também encontrou relação entre as diferentes estruturas de valores, embora tenha utilizado outro modelo teórico e outra técnica de análise de dados. A semelhança encontrada entre os resultados dos dois estudos dá mais suporte para a necessidade de que as correntes de estudo de valores laborais e as de valores pessoais gerais sejam integradas. Essa integração, provavelmente, poderá aumentar a compreensão dos antecedentes e consequientes do fenômeno, além de propiciar o desenvolvimento de modelos teóricos mais sólidos para compreender os valores.

O modelo teórico de valores pessoais gerais de Schwartz (2005) foi adequado para compreender os valores laborais, exceto no que se refere às relações de conflito da dimensão Abertura à Mudança e Conservação com a dimensão Realização Profissional e Estabilidade. É possível que, dado o contexto social em que se insere a amostra, as relações de conflito não são percebidas. Além disso, é importante ressaltar que existe uma limitação na análise de dados que pode ter influenciado esse resultado. Não foi possível utilizar o método de estimação Satorra-Bentler, que é apontado por Fouladi (2000) como o mais efetivo no caso de variáveis não- 
normais, porque o programa utilizado, AMOS, não realizava esse tipo de análise. O uso dessa técnica é relevante para a análise desses dados que apresentaram considerável desvio da normalidade.

Com relação ao modelo teórico utilizado, vale lembrar que ele não aborda a dimensão "esforço corporal e desumanização", proposto por Borges (1999). Estudos futuros deveriam testar o modelo teórico da autora e identificar a pertinência da inclusão dessa dimensão para compreender os valores laborais de indivíduos provenientes de diferentes classes socioeconômicas.

\section{Referências}

Bollen, K. A. (1989). Structural equations with latent variables. Nova York: John Wiley and Sons.

Borges, L. O. (1998). Significado do trabalho e socialização organizacional: Um estudo empírico entre trabalhadores da construção habitacional e de redes de supermercados. Teses de doutorado, Universidade de Brasília, Brasília.

Borges, L. O. (1999). A estrutura fatorial dos atributos valorativos e descritivos do trabalho: um estudo empírico de aperfeiçoamento e validação de um questionário. Estudos de Psicologia, 4, 107-139.

Elizur, D. (1991). Work and nonwork relations: The conical structure of work and home life relationship. Journal of Organizational Behavior, 12, 21-38.

Elizur, D. \& Sagie, A. (1999). Facets of personal values: A structural analysis of life and work values. Applied Psychology: An International Review, 48, 73-87.

Fouladi, R. T. (2000). Performance of modified test statistics in covariance and correlation structure analysis under conditions of multivariate nonnormality. Structural equation modeling, 7, 356-410.

Gouveia, V. V., Martínez, E., Meira, M. \& Milfont, T. L. (2001). A estrutura e o conteúdo universais dos valores humanos: Análise fatorial confirmatória da tipologia de Schwartz. Estudos de Psicologia, 6, 133-142.

Guttman, L. (1968). A general nonmetric technique for finding the smallest coordinate space for a configuration of points. Psychometrika, 33, 469-506.

Kinnane, J. F. \& Gaubinger, J. R. (1963). Life values and work values. Journal of counseling Psychology, 10, 362-367.

Kline, R. B. (1998). Principles and practice of structural equation modeling. New York: Guilford Press.

Little, R.J. \& Rubin, D.B. (1987). Statistical Analysis with Missing Data. New York: Wiley \& Sons.

Mardia, K. V. (1971). The effect of nonnormality on some multivariate tests and robustness to nonnormality in the linear model. Biometrika, 58, 105-121.

Ministério da Educação e Cultura do Brasil (2002). Diretrizes curriculares para os cursos de graduação [on-line]. Retirado em 05/11/2002 de www.mec.gov.br/sesu/diretriz.htm
Osborne, J. (2002). Notes on the use of data transformations. Practical Assessment, Research \& Evaluation, 8(6). Retirado em 08/09/2005 http://pareonline.net/getvn.asp?v=8\&n=6.

Porto, J. \& Tamayo, A. (2003). Desenvolvimento e validação da escala de valores do trabalho - EVT. Psicologia Teoria e Pesquisa, 19, 145-152.

Porto, J. B., Ros, M. \& Tamayo, A. (2003). Escala de Valores Laborales: Un estudio de equivalencia de estructuras [Resumo]. Em Sociedade Interamericana de Psicologia (Org.). Resúmenes, XXIX Congresso Interamericano de Psicologia (p.75). Lima - Peru: Sociedade Interamericana de Psicologia.

Ros, M., Schwartz, S. H. \& Surkiss, S. (1999). Basic individual values, work values, and the meaning of work. Applied Psychology: An International Review, 48, 49-71.

Sagie, A. \& Elizur, D. (1996). The structure of personal values: a conical representation of múltiple life areas. Journal of organizational behavior, 17, 573-586.

Schiffman, S. S., Reynolds, M. L. \& Young, F. W. (1981). Introduction to multidimensional scaling. New York: Academic Press.

Schwartz, S. H. (1992). Universals in the content and structure of values: Theoretical advances and empirical tests in 20 countries. Em M. P. Zanna (Org.), Advances in experimental social psychology, vol. 24. (pp. 1-65). San Diego: Academic.

Schwartz, S.H. (2005). Valores Humanos Básicos: seu contexto e estrutura intercultural. Em A. Tamayo; J.B. Porto (Org.), Valores e comportamento nas Organizações (pp. 21-55). Petrópolis: Vozes.

Schwartz, S. H. \& Bilsky, W. (1987). Toward a universal psychological structure of human values. Journal of Personality and Social Psychology, 53, 550-562.

Schwartz, S. H. \& Bilsky, W. (1990). Toward a theory of the universal content and structure of values: Extensions and Cross-Cultural Replications. Journal of Personality and Social Psychology, 58, 878-891.

Schwartz, S. H. \& Boehnke, K. (2004). Evaluating the structure of human values with confirmatory factor analysis. Journal of Research in Personality, 38, 230-255.

Shye, S., Elizur, D. \& Hoffman, M. (1994). Introduction to facet theory: content design and intrinsic data analysis in behavioral research. Thousand Oaks: Sage.

Tamayo, A. \& Schwartz (1993). Estrutura motivacional dos valores. Psicologia: Teoria e Pesquisa 9, 329-348.

Ullman, J. B. (2001). Structural equation modeling. Em B. Tabachnick. \& L. S. Fidell (Orgs.). Using Multivariate Statistics ( $4^{\mathrm{a}}$ ed.). San Francisco: Allyn \& Bacon.

Recebido em 12.08.2005

Primeira decisão editorial em 09.03.2006

Versão final em 21.08.2006

Aceito em 09.10.2006 